nearly resemble the last which I have just written, that it would be us. less to transcribe them.

CASE 7.- A woman, about thirty-five years of age, who had experienced for some time constant pain in her groins, and a mucous discharge from the vagina, whose stomach was weak, and who was much distressed by hysterical symptoms, consulted a practitioner, who recommended that she should take five grains of pilul. hydrargyrii at bedtime, and twice in the course of the day a cupful of chamomile tea. She pursued this plan for more than a fortnight, at the end of which time she become worse, the discharge increased, and the hysterical feelings were unabated. When the author was consulted, the seat of the disease induced him to make an examination, when there was found a thickened state of the cervix of the uterus, which was tender to the touch. A horizontal posture, the injection of tepid water into the vagina, and small doses of saline purgatives, after some time, relieved the complaints, which were only symptomatic of the disease of the nterus. - " Observations on those Diseases of Females attended by Discharges," vol. i. p. 219.

CASE 8. - It is many years since my friend, the late Dr. Olive, took me with him into the city, to see a person with an affection of the uterus; she was about forty-two, married, but had had no children. She was emaciated, and the skin had that peculiar appearance characteristic of malignant disease; there was a mucous discharge from the vagina, and sometimes, upon any unusual exertion or mental anxiety, copious discharges of blood; the mucous discharge, too, was occasionally tinged with blood; and at each catamenial period the catamenial discharge was more copious than usual, and mixed with coagula. She complained of lancinating pairs through the pelvis. By an ordinary examination per vaginam I found the cervix uteri indurated and enlarged, and when pressure was made upon it she complained of a very acute lancinating pain; the os uteri was enlarged, with its borders everted and uneven, and almost of the hardness of cartilage This was the first case of carcinoma of the uterus I ever saw. had, a few weeks before, been reading the work of Sir Charles Clarke, and from his graphic descr ption of this state of the os uteri in cases of carcinoma, I told $\mathrm{mr}$ friend that $\mathrm{I}$ thonght this was a case of carcinoma. She died in six months, and at the post-mortem examination it was found that the ulceration had spread from the uterus to the bladder and rectum, which parts it had perforated, forming the pelvis into a reserroir, in which were received the urine, fæces, and the discharge from the diseased surface.

CAss 9.-C. H-, a widow, about 35, who had had a family, the youngest of which was six years old, for the last six months had the catamenia more frequently than usual; the discharge was more copious, and mixed with coagula hetween the periods she had sometimes a purulent discharge, and occasionally a discharge of pure blood from the vagina; she complained, too, of violent lancinating pains in the situation of the uterus, and a constant dull pain occupying the sacrum. She felt a constant nausea, but did not vomit unless she took food, and then rarely. There was considerable tendemess experienced at the lowest part of the abdomen in the region of the bladder. Upon an examination per vaginam, I found the cervix uteri enlarged and indurated; excruciating pain of a lancinating character was felt when I pressed upon it. I could feel the cervix uteri uneven and rough, and upon using the speculum, an ulcer could be seen which occupied the anterior lip of the os uteri; the posterior was already destroyed. In this case the os uteri was not more open than in its normal condition. She died about eight months after I first saw her, and at the post mortem examination, no one conld doubt the real nature of the case, that it was a case of carcinoma.

CaSE 10.- Of the following case I made notes, about eleven years since, at the time I saw the patient, a married lady, aged 38 , who had had a family, the youngest of which was ten years old. She stated, that for the last six months she had experienced a dull aching pain in the back, extending down the thighs; there was uneasiness in the groins, and a discharge of a purulent appearance; she had uneasiness about the region of the bladder, and a frequent desire to make water; the uneasiness and pain were increased in the evening, and not relieved by the recumbent posture, at which time she generally became feverish; her appetite was bad, and her bowels were confined, and when they acted, she suffered considerable pain. By an examination per vaginam, I found the os uteri patulous, but its labia were not everted, nor were their surfaces uneven, but smooth. and soft. This was clearly a case of inflammation of the uterus; and the recumbent position, local bloodletting, by cupping and leeches to the os uteri, were advised, with saline purgaiives and a warm hip-bath, with injections of warm water into the vagina. Under this p'an of treatment her general health was considerably improved, and at that time 1 left England for a considerable period. and my patient lost her contidence in the plan of treatment which she had.been pursuing. She no longer abstained from exercise, and abandoned every other remedy which had been prescribed for her, and her complaint soon returned. She now consulted an eminent physician in the City, who told her it was incipient cancer. I last week received a letter from her, and she tells me she is quite well.

\section{ON THE TREATMENT OF FEMORAL HERNIA.}

By J. Sebastian Wilkinson, Esq.,Surgeon, London.

THE following case of femoral hernia occurred to me in the course of my practice some years ago; and having met with similar cases since, in the treatment of which $I$ have been equally successful, I beg the favour of its insertion in the widely-circulated journal, THE LANCET.

Mrs. Wto $m e$ in the early part of the spring of 1833, to know if $I$ could afford her any relief, as her case was pronounced irreducibie and incurable. The subjoined is her own history of the case:-

"The swelling in the groin first appeared in the year 1823. It conld then be easily returned into the abdomen. I thought nothing of it, and neglected to apply a truss. About four years before I applied for medical advice, I could not return the swelling. It was occasionally painful, especially when the bowels were confined. About three months before I consulted you, I became alarmed, owing to the increased size of the tumour, and the pain I experienced in walking. I was obliged to be particular in my diet, and keep the bowels always relaxed. I then lived as cook and housekeeper in a family residing at Newport Pagnell, who called in their family surgeon. He said he could do nothing for me, but sent me to London to Mr., now Sir Benjamin Brodie. This gentleman examined me, and said it was quite irreducible and incurable, and that my life would be endangered by any blow upon the part, or from inflammation arising from walking. He, however, considered it advisable to wear a piece of thick leather, beat out in the form of a cup, over the tumour, to be confined by a strap round the lower part of the body."

When I saw the patient, the tumour was as big as a large walnut, doubled over Poupart's ligament; moveable, but confined at the femoral ring. It consisted of intestine and omentum, and quite irreducible. Having observed in the dissecting-room, subjects with old herniæ, with both abdominal and crural rings of a large size, I considered it feasible that dilatation might gradually be accomplished in an inverse direction.

The patient being very fat, I first reduced her in substance by bleeding twice a week, to eight, and afterwards to five ounces low diet, consisting of broth and gruel, with warm baths, three times a-week, and occasional doses of castor oil. When the skin had become flabby, and her size reduced, I used daily manipulations, pressing the tumour downwards and then upwards. In this way $I$ proceeded for nearly six months, and at last succeeded in returning the rupture. I tied my silk handkerchief in a large knot, which I placed in the groin of the patient, and confined the ends on the opposite side of the pelvis, thus affording a temporary truss. I put her into a coach and sent her to $\mathbf{M r}$ Brodie, who returned me the following note:-

"Mr Dear Sir,-There appears to be nothing left but the sac, and probably a small portion of adhering omentum. There can be no objection to the patient wearing one of Salmon and Ody's trusses.

"J. S. Wilkinson, Esq."

Mrs. W- soon afterwards got married, and is now living in good health, with her husband, who is a farmer in Herefordshire.

Dcrset-street, Manchester-square, Nov. 7, 1344

\section{ON THE LABOURS OF GRAHAMIZED DOCTORS IN} THE COUNTRY.

By R. Hodgson, Esq. Surgeon, Clapham, Yorkshire.

Seexag the editor of The Lancer so landably and strenu. ously exerting himself in belialf of the medical profession, and in opposition to Sir James Grabam's quack protection Bill, $\mathbf{I}$ think it the duty of every medical practitioner to endeavour to open the eyes of the public to the dire results which may be expected from the operation of such a bill; I will therefore state a few cases which have occurred in this neighbourhood, under the slight protection of the present law. What, then, must be the dreadful conseguences to the community when all restriction is abolished?

In the year 1830, a fellow, called Hubhard, better known by 
the soubriquet of the Blind Doctor, was sent for to a respectable married woman, about fifty years of age, labouring under an attack of paraplegia; her general health was good up to this time. This bright genius proclaimed effecting a cure in a fortnight; in fact, he promised in that time she would be able to rival Taglioni in the dance.

He commenced his curative process by violent frictions on the lumbar region, the nates, and back part of the thighs, with the following embrocation:-Strong spirit of ammonia, turpentine, and landanum, of each equal parts; and, previous to his manipulations, he stripped his coat, and to make it appear the occupation was laborious, he stipulated to be allowed two glasses of strong grog during the friction. Things went on in this way for about a month, when I was called in to see her, and, lo! a horrible spectacle was presented; the female, who one short month before was lusty, hale, and healthy, was now emaciated, pale, fretful, and care-worn; the lumbar region, nates, and thighs presented one mass of mortification and corruption, and she sunk in less than a week, notwithstanding the use of tonics, sedatives, and all means that could be devised.

The second case was a young female with anchylosed knee. His treatment in this case was somewhat different; in addition to the friction the patient sat upon a table, with the knee forcibly bent over its edge, and she had suspended to her foot a fifty-six pound weight for an hour each day; the consequence was violent inflammation, with much constitutional irritation, but with rest and active antiphlogistic treatment this individual slowly recovered.

I could record more cases, but these are enough to shew the extreme danger of this quack's practice. When questioned about the modus operandi of his application, his answer was, "The ammonia opens the pores, the turpentine brings the disease to the skin, and the laudanum drives it away." And will it be believed, that in a small village, during his short stay, the fellow was re. ceiving $20 l$. or $30 l$. or upwards per week. He told me his charge for a small phial of the liniment was $17 s .6 d ., 1 l .10 s .6 d$, , or 2l. $0 s .6 d$. He charged as he thought his dupes were able to pay, and he shrewdly observed, that he always added a sixpence or threepence to the charge, to make it appear he did not impose upon them. So much for the Blind Doctor.

There is another quack in this neighbourhood, by trade a weaver, who was consulted, a few years ago, in a case of aneurism in the popliteal, which was proclaimed by him a blast; consequently, he tried to make it ripe, as he termed it, by poultices, \&c.; the result was, the aneurism burst, and the poor person died in his presence from hæmorrhage, without his being at all aware of the cause of the bleeding. It is useless to add other similar fatal facts, but I trust these will shew to any reflecting mind the necessity of much greater protection to the public than at present exists, whatever Hudibrastic pleasure that public may have in being cheated.

Nov. 6, 1844.

\section{BRITISH MEDICAL JOURNALS.}

\section{STATISTICS OF OBSTETRIC PRACTICE.}

The Origin of Puerperal Fever.-As tending to illustrate this most disputed question, Dr. Murphy relates a case of difficult and protracted labour, in which delivery was finally effected by perforation. It was necessary to remove (by introducing the hand) the retained placenta. On the fifth day the patient presented symptoms of puerperal fever, and died in four days. A postmortem examination was not allowed. The author remarks-

" Every practical accoucheur is aware, that the discharges from the uterus, arising from decomposition of the membranes, are often of a very acrid nature. The yellow oily discharge which flows sometimes abundantly from this organ after a longcontinued labour, or where the child is putrid, is known to cause, if caution be not exercised, a great deal of irritation of the skin; furuneuli and impetiginous eruptions have been the result. But before the present instance the writer has never met with a case in which the discharge on the surface of the uterus acted as a morbid poison. In this case, it being necessary to withdraw the placenta, each arm was successively passed into the uterus, and both were grasped tightly by its cervix. Pustules appeared on the arms two days after this, which at first seemed to be similar to those produced by the acrid discharges of the uterus. One of them, however, soon presented new characters. A deeply livid base surrounded the pustule, and that part of the arm near it was becoming hard and swollen. The promptitude with which a judicious treatment was carried into effect, prevented much constitutional disturbance taking place. This pustule had all the appearance of the " pustule maligne," or " charbon," which is well known to occur in the South of France. It prevails epidemically among horned cattle, and is derived from their blood by the butchers, with whom the pustule is often observed.

The argument from analogy in the present case seems to be, that a morbid poison was generated in the blood of the patient, (epidemically?) contact with which was sufficient to communicate the taint, and to convert an ordinary furunculus into a malignant pustule; and that, by parity of reasoning, the same altered condition of the blood caused all the phenomena of puerperal fever in the patient. This view receives a stronger support from the fact, that the patient was free from the ordinary symptoms of uterine inflammation, which were anxiously looked for as the danger expected to arise."

We must leave Dr. Murphy's views to the consideration of our readers. The space which we have afforded to the analysis of his communication attests our opinion of its merits. We should have mentioned, that it appears as a report of the author's practice at University College Hospital during a period of two years.

ON THE USE OF NITRIC ACID AS AN ESCHAROTIC IN CERTAIN HAMORRHOIDAX AFFECTIONS.

In a previous number of the same (Dublin) journal, Dr. Houston makes the following remarks on this subject:-

"The failures which have occurred in other hands may, perhaps, be often traceable to want of discrimination as to the fitness of the case for the remedy, or to some imperfection in the application of the latter. As I have known errors of this kind to happen, I must repeat, that the only case which the nitric acid will serve, is the internal bleeding pile-that soft, red, strawberrylike elevation of the mucous membrane, for which I have used the term vascular tumour, and which the acid removes by the production of a slough of its surface. The surface to be acted upon must be soft, and free from any coating of cuticle, such as is apt to form on it by persistent prolapse; for if the acid be used in a case so circumstanced, nothing more than a removal of the cuticle may be expected from the application; and further, to ensure to the caustic its full effect, the part to be touched should, beforehand, be dried and cleared of all mucous or other adherent fluids. There is no danger, that I know of, to be apprehended from the application of the acid. The acid must be laid on in quantity, and rubbed in with force enough to be pressed into the pores of the surface, [and the tumour must, if possible, be returned within the sphincter.] At the best, the caustic produces only a very superficial slough; and, on this account, it will be necessary, in some cases - as where the tumours are old and firm in texture-to make a second, and even a third, application."

These remarks are followed by a series of cases illustrating the author's views. He then further observes, that the remedy is not so painful in its application as it may at first sight appear to be. In its action, the acid, by destroying the vascular surface, arrests the hæmorrhage, and if the relaxation be not immoderate, cures the prolapse by bracing up the textures underneath.

The treatment further consists of attention to diet, to cleanliness, and the use of remedies, local or general, calculated to remove the primary exciting cause of the disease.

From some recent numbers of the Provincial Journal which are before us, we extract the few following matters of interest:-

\section{DIFFICULTY IN RECOGNISING THE SEX OF AN INFANT.}

Mr. Terry, of Northampton, was requested to examine the genital organs of an infant believed to be, and baptized as, a female. On a first examination, the case appeared to be one of imperforate vagina. The infant, however, proved to be a male, whose genitals were somewhat retracted, and covered by loose folds of integument and cellular tissue; on turning these aside, the penis and its appendices were exposed. The orifice of the urethra presented itself behind the glands at the frænum, thus constituting hypospadias. The mother's surprise, and fear of the jokes of her friends at her inability to tell the sex of her infant, may readily be conceived.

\section{CASES OF FRACTURED SKULL.}

Mr. Thomas exhibited to the Sheffield Medical Society the skull of a child who had been trampled by a horse. During life, the appearances of iujury were confined to puffiness of the scalp. After death, "a very considerable depressed fracture" of the parietal bone, lacerating the lateral sinus, was discovered. 